\title{
Optimisation thermique du transistor à haute mobilité d'électron (HEMT) par la méthode CMA-ES
}

\section{Thermal optimization of the high electron mobility transistor (HEMT) using the CMA-ES method}

\author{
Amar Abdelhamid ${ }^{1}$, Hamdani Hamid ${ }^{2}$, Radi Bouchaïb ${ }^{3}$, El Hami Abdelkhalak ${ }^{4}$ \\ ${ }^{1}$ Laboratoire LIMII, FST, Hassan First University of Settat, Maroc, amarabdelhamid20@gmail.com. \\ ${ }^{2}$ LIMII, FST, Hassan First University of Settat, Maroc, hamidelhamdani@gmail.com. \\ ${ }^{3}$ Laboratoire LIMII, FST, Hassan First University of Settat, Maroc, bouchaib.radi@yahoo.fr. \\ ${ }^{4}$ Laboratoire LMN, INSA, Rouen, France, Abdelkhalak.elhami@insa-rouen.fr.
}

\begin{abstract}
RÉSUMÉ. L'optimisation vise à assurer une conception robuste des systèmes avec un cout minimal, ce travail s'intéresse à l'optimisation du transistor à haute mobilité d'électrons (HEMT), il est un élément très important dans les systèmes mécatroniques à haute puissance. II contient dans sa structure plusieurs couches de matériaux, les paramètres géométriques et thermiques de ces couches influencent la température de fonctionnement du transistor, d'où sur sa performance. En utilisant la méthode de CMA-ES codée sur Matlab, un modèle éléments finis développé sur Comsol multiphysics et un couplage entre les deux logiciels, on a optimisé ces paramètres afin de réduire la température maximale du fonctionnement du HEMT, pour que le transistor assurent sa fonction avec moins d'influence sur les autres caractéristiques.

ABSTRACT. The optimization aims to ensure a robust system design with minimal cost, this work focuses on the optimization of the High Electron Mobility Transistor (HEMT), it is a very important element in high power mechatronic systems. It contains in its structure several layers of materials, the geometrical and thermal parameters of these layers influence the operating temperature of the transistor, hence its performance. Using the CMA-ES method coded on Matlab, a finite element model developed on Comsol multiphysics and a coupling between the two softwares, these parameters have been optimized in order to reduce the maximum operating temperature of the HEMT, so that the transistor performs its function with less influence on the other characteristics.

MOTS-CLÉS. optimisation, HEMT, CMA-ES, systèmes mécatroniques.

KEYWORDS. optimization, HEMT, CMA-ES, Mecatronics systems.
\end{abstract}

\section{Introduction}

Au cœur des systèmes mécatroniques, le transistor joue un rôle très importent, car il assure plusieurs fonctions comme la commande, l'amplification et la minimisation des signaux. Parmi ces transistors on trouve le transistor à haute mobilité d'électron (HEMT) à base de nitrure d'aluminiumgallium/nitruregallium $(\mathrm{AlGaN} / \mathrm{GaN})$, c'est un composant électronique très puissant grâce à sa structure et ses matériaux [ELH 17]. Ce transistor a été utilisé dans plusieurs applications à haute fréquence et à haute température [GAR 16][WAN 18], tels que la télécommunication, la guerre électronique (domaine militaire) et les systèmes aéroportés[DYJ 18][DON 19]. Le HEMT a été utilisé aussi dans plusieurs systèmes tels que les amplificateurs à haute puissance, les radars et les satellites, il se trouve aussi dans les capteurs et les dispositifs à radiofréquence [CHE 19]. En plus, la température de fonctionnement est un facteur très important, elle influence la fiabilité du HEMT, car la plupart de ses caractéristiques telle que la mobilité des électrons, la conductivité thermique [DAS 06], la vitesse de saturation et autres sont en fonction de la température [JIA 18][BEL 19]. Toutes ces caractéristiques ont tendance à se dégrader avec l'élévation de la température à cause de phénomène de l'autoéchauffement du transistor [RAD 19][SAM 20]. Par conséquent ça donne naissance à des dégradations comme l'enterrement de la grille, endommagement de la connexion entre la puce et le boitier [WAN 18], la dégradation de la mobilité des électrons et la réduction de courant [ALI 20]. Dans le cas des systèmes aéroportés, la majorité des défaillances sont causés par des amplificateurs à haute puissance 
au niveau de ses transistors à haute puissance, ces défaillances sont à cause de la température de fonctionnement des transistors qui influence négativement leur fiabilité et donc celle de système [BAC $15 \mathrm{a}]$.

Pour assurer le bon fonctionnement du HEMT, il est nécessaire d'améliorer son comportement thermique, pour que sa température ne dépasse pas des valeurs critiques. Par conséquent, il faut penser à diminuer la température maximale du fonctionnement du HEMT D'autre part, l'optimisation de dimensionnement sert à trouver les paramètres de la conception qui minimise une fonction objective. Dans ce sens CMA-ES (Covariance Matrix Adaptation-Evolution Strategy) est une méthode de recherche stochastique basée sur la population dans des espaces continus et discrets [HAN 16], elle sert à résoudre des problèmes d'optimisation par la minimisation d'une fonction objective, la fonction objective à minimiser est la température maximale de fonctionnement du transistor. Cette méthode a montré une efficacité à travers des résultats obtenus dans plusieurs travaux qui vise la fiabilité des systèmes [ELH 15].

Dans un premier temps, on va faire une aperçue sur le HEMT : structure, avantages et utilisations. Par la suite on va développer un modèle thermique permettant de simuler le comportement thermique du HEMT et identifier les paramètres à minimiser. Ensuite on définit la méthode CMA-ES, ses paramètres et son algorithme. Dans la partie suivante, un couplage entre les deux logiciels Matlab et Comsol multiphysics sera développé, il permet de connecter les deux modèles thermique et statistique afin de résoudre le problème de l'optimisation. A la fin on extrait les résultats et on fera une comparaison de la variation de la température de fonctionnement du HEMT avant et après l'optimisation.

\section{Problème de l'optimisation}

Le transistor à haute mobilité d'électron est un composant électronique très important dans les systèmes mécatroniques, il assure plusieurs fonctions comme l'amplification, la diminution et la commande des signaux. Grâce à ses caractéristiques électriques et thermiques, le HEMT est utilisé dans plusieurs domaines tels que les systèmes aéroportés surtout au niveau des amplificateurs à haute puissance, l'automobile, la télécommunication et la guerre électronique[DJ 18][DON 19].

En plus, ce transistor possède une structure très spéciale, il est composé de plusieurs couches de différents matériaux, souvent il est construit sur un substrat en $\mathrm{SiC}$ (carbure de silicium), puis une couche de nucléation séparant le substrat et la couche de GaN, une couche en AlGaN sert créer une hétérojonction avec la couche de GaN pour produire le gaz 2DEG qui représente le canal des électrons [AMA 19]. Les propriétés de ces matériaux ainsi que les caractéristiques du HEMT sont en fonction de la température du fonctionnement. La conductivité thermique, la mobilité des élections, la vitesse de saturation et autres propriétés ont tendance à se dégrader avec l'augmentation de la température du fonctionnement du transistor, cette température peut atteindre des valeurs critiques à cause de phénomène de l'auto-échauffement [RAD 19][SAM 20]. On constate que l'élévation de la température influence la fiabilité et la performance du HEMT, d'où la nécessité de chercher à diminuer cette température pour augmenter la fiabilité. Pour réaliser ce but, des méthodes seront utilisés comme la méthode des éléments finis et la méthode CMA-ES.

\section{Modèle éléments finis}

Lors de son fonctionnement, Le HEMT dissipe une puissance (Pdiss) au niveau de la zone active par le phénomène de l'auto-échauffement, cette puissance est en fonction de la tension (Vds) et le courant (Ids) qui circule entre le drain et la source, elle a comme expression [BAC 15b] : 


$$
\mathrm{P}_{\mathrm{diss}}=\mathrm{V}_{\mathrm{ds}} * \mathrm{I}_{\mathrm{ds}}
$$

Afin d'étudier l'effet de la puissance dissipe sur le comportement thermique du HEMT, une modélisation thermique est nécessaire, cependant, il faut étudier le transfert thermique au sein du transistor, le mode du transfert principale est la conduction et l'équation de chaleur sera [ELH 15] :

$$
k \cdot \nabla^{2} T=\rho \cdot C_{P} \cdot \frac{\partial T}{\partial t}+Q
$$

Avec :

-Q power dissipated in joules;

- K conductivité thermique $(\mathrm{w} / \mathrm{m} / \mathrm{k})$;

- $\rho$ density Kg. $\mathrm{m}^{-3}$;

- Cp capacité massique thermique $\left(\mathrm{J} \mathrm{Kg}^{-1} \cdot \mathrm{K}^{-1}\right)$;

- $\mathrm{T}$ température $(\mathrm{K})$

Cette modélisation sera mise en place en utilisant la méthode des éléments finis, elle permet de résoudre l'équation de chaleur et d'observer l'influence des paramètres thermique et géométrique sur le comportement thermique du HEMT. Les propriétés des matériaux utilisés dans la modélisation : la conductivité thermique, le densité volumique, la capacité thermique massique avec leurs valeurs, sont

\begin{tabular}{|c|c|c|c|}
\hline Matériaux & $\rho[\mathrm{kg} / \mathrm{m} 3]$ & $\mathrm{KTH}[\mathrm{W} / \mathrm{m} / \mathrm{K}]$ & $\mathrm{Cp}$ [ J/kg/K ] \\
\hline $\mathbf{A u}$ & 19300 & 310 & 137 \\
\hline SiN & 3300 & 10 & 713 \\
\hline AlGaN & 5470 & $\left.25 \cdot \frac{293}{273+T}\right)^{1,35}$ & 548 \\
\hline GaN & 6100 & $\left.161 \cdot \frac{293}{273+T}\right)^{1,45}$ & 490 \\
\hline $\mathrm{SiC}$ & 3220 & $\left.416 \cdot \frac{293}{273+T}\right)^{1,5}$ & 690 \\
\hline AIN & 6100 & $\left.6,7 \cdot \frac{293}{273+T}\right)^{2,7}$ & 490 \\
\hline
\end{tabular}
mentionnées dans la tableau 1 [JAR 14][CHE 13] :

Tableau 1. Propriétés des matériaux

Le modèle élément fins est développé sur le logiciel éléments finis Comsol multiphyscs, il permet d'étudier l'influence de plusieurs paramètres du HEMT sur sa température du fonctionnement, la figure 22 montre l'influence de la puissance dissipée (représentant l'un des condition du fonctionnement) sur la variation de la température de HEMT [AMA 19].

La modélisation thermique du transistor par la méthode des éléments finis (MEF) a permis d'identifier les paramètres de la géométrie, qui ont un grand impact sur la température de HEMT. L'épaisseur du substrat (a), l'épaisseur de la couche de nucléation (b) et le développement du transistor (c), sont les trois paramètres (en $\mu \mathrm{m}$ ) qui ont une grande influence sur le comportement thermique du 
transistor. Ce modèle éléments finis sera Le but de l'optimisation dans ce cas est de minimiser la température maximale du fonctionnement du transistor.

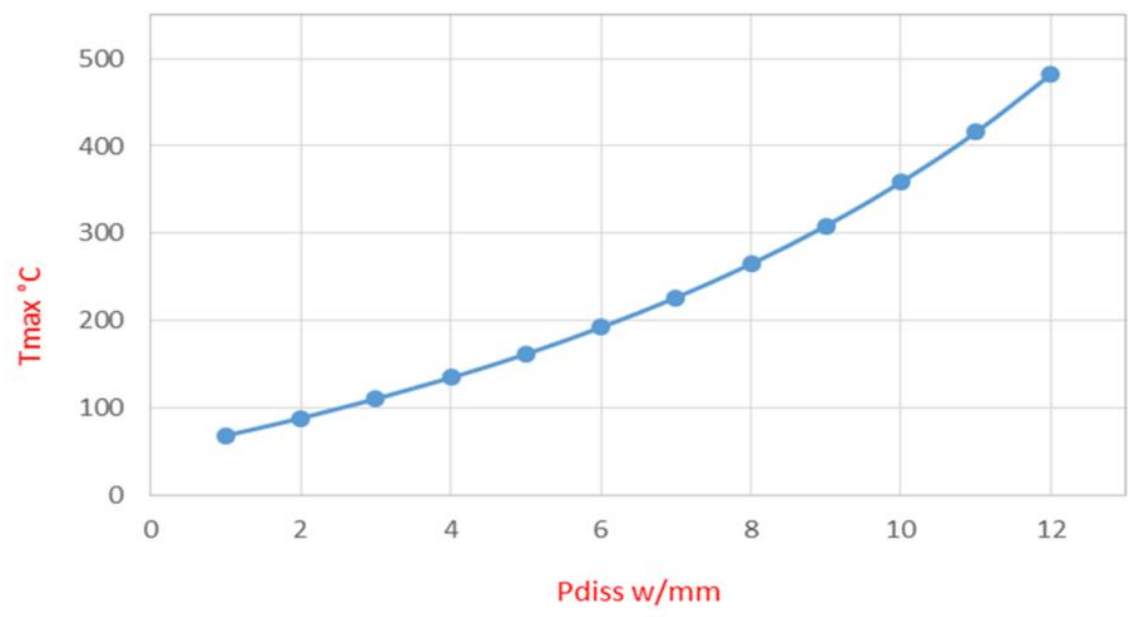

Figure 1. Evolution de la température en fonction de la puissance dissipée

\section{Covariance Matrix Adaptation-Evolution Strategy ( CMA-ES )}

La méthode CMA-ES(Covariance Matrix Adaptation-Evolution Strategy) est le meilleur membre de la famille des algorithmes d'évolution, est une méthode d'optimisation stochastique sans dérivée et peut s'adapter à des problèmes d'optimisation non convexe, CMA-ES est algorithme d'optimisation plus puissant par rapport aux autres, elle est basée sur les quatre principes de la sélection naturelle : évolution, sélection, recombinaison et mutation [SIM 14]. La recombinaison des éléments (parents) d'une population génère de nouveaux individus (enfants), les nouveaux individus subissent une mutation pour qu'ils deviennent des nouveaux membres de la même population, afin de garder et adapter la taille de population une sélection est faite en choisissant les meilleurs individus après la mutation. La mise en place des opérations évolutionnaires assure cette démarche, pour guider la démarche vers un optimum globale dans un espace de recherche continu ou discret [HAM 19a]. CMAES $(\lambda, \mu)$ est basé sur l'adaptation de la matrice de covariance de la oi multi normalednas $R^{n}$, cette adaptation est équivalent de la construction d'une approximation de la fonction objectif $\mathrm{f}$, cette méthode génère 1 nouveaux individu a partir de mu éléments de la population. CMA-ES $(\lambda, \mu)$ génère la population en échantillonnant une distribution multinormale [SIM 14][HAN 17] :

$$
\boldsymbol{x}_{k}^{(g+1)} \sim \boldsymbol{m}^{(g)}+\sigma^{(g)} \mathcal{N}\left(\mathbf{0}, \boldsymbol{C}^{(g)}\right) \quad \text { fork }=1, \ldots, \lambda
$$

avec :

indique la même répartition à gauche et à droite.

$\mathcal{N}\left(\mathbf{0}, \boldsymbol{C}^{(g)}\right)$ est une distribution normale multivariée avec une moyenne nulle et une matrice de covariance $\mathrm{C}(\mathrm{g})$.

$\mathrm{x}_{k}^{(g+1)} \in \mathbb{R}^{n}, k$ ième progéniture (individu, point de recherche) de la génération $\mathrm{g}+1$.

$\mathrm{m}(\mathrm{g}) \in \mathrm{Rn}$, valeur moyenne de la distribution de la recherche à la génération $\mathrm{g}$.

$\sigma(\mathrm{g}) \in \mathrm{R}>0$, la taille de pas, à la génération $\mathrm{g}$. $\mathrm{C}(\mathrm{g})$

$\in \mathrm{Rn} \times \mathrm{n}$, matrice covariance à la génération $\mathrm{g}$.

$\lambda \geq 2$, la taille de la population. 
Par suite, les images des individus générés par échantillonnage sont évaluées par la fonction objectif, les meilleurs individus seront regroupés dans le vecteur moyen $\mathrm{m}$, il a comme expression :

$$
\boldsymbol{m}^{(g+1)}=\sum_{i=1}^{\mu} w_{i} \boldsymbol{x}_{i: \lambda}^{(g+1)}
$$

et

$$
\sum_{i=1}^{\mu} w_{i}=1, \quad w_{1} \geq w_{2} \geq \cdots \geq w_{\mu}>0
$$

avec :

$\mu \leq \lambda$ le nombre des points sélectionnés ou la taille de la population originale (parents).

$w i=1 \ldots \mu \in \mathrm{R}>0$, représente les pois de recombinaison, sont strictement positifs et vérifiant l'équa1 [5] [5]. xi: $\lambda$ : le iene meilleur individu sélectionné de l'ensemble des individus $[\mathrm{x} 1, \mathrm{x} 2, \cdots, \mathrm{x} \lambda]$ générés par l'équation [3].

$\mathrm{i}: \lambda$ est l'indice du ieme individu après classement tel que, $\mathrm{f}(\mathrm{x} 1: \lambda) \leq \mathrm{f}(\mathrm{x} 2 \cdot \lambda) \leq \cdots \leq \mathrm{f}(\mathrm{x} \lambda: \lambda)$, où $\mathrm{f}$ est la fonction objectif.

Après la mise à jour du vecteur moyen $\mathrm{m}$, l'étape suivante est l'adaptation de pas $\sigma$ et la matrice de covariance C.la mise à jour des deux paramètres se fait par deux chemins d'évolution pc et $\mathrm{p}_{\sigma}$ [24]. Cependant, la suite du processus de la méthode CMA-ES nécessite d'actualiser le chemin d'évolution $\mathrm{p}_{\sigma}$ permettant de mettre à jour la taille du pas $\sigma$, les deux paramètres se calculent suivant les équations suivantes :

$$
\begin{aligned}
\mathbf{p}_{\sigma}^{(g+1)} & =\left(1-c_{\sigma}\right) \mathbf{p}_{\sigma}^{(g)}+\sqrt{c_{\sigma}\left(2-c_{\sigma}\right)} \sqrt{\mu_{w}}\left(\mathbf{C}^{(t)}\right)^{-\frac{1}{2}} \frac{\mathbf{m}^{(g+1)}-\mathbf{m}^{(g)}}{\sigma^{(t)}} \\
& =\left(1-c_{\sigma}\right) \mathbf{p}_{\sigma}^{(g)}+\sqrt{c_{\sigma}\left(2-c_{\sigma}\right)} \sqrt{\mu_{w}} \sum_{i=1}^{\mu} w_{i} \mathbf{z}_{i \lambda}
\end{aligned}
$$

$\sigma^{(g+1)}=\sigma^{(g)} \exp \left(\frac{c_{\sigma}}{d_{\sigma}}\left(\frac{\left\|\mathbf{p}_{\sigma}^{(g+1)}\right\|}{\mathbb{E}\left(\left\|\mathcal{N}_{d}(\mathbf{0}, \mathbf{I})\right\|\right)}-1\right)\right.$

$p_{\sigma}^{(g)} \in \mathbb{R}^{d} \quad$ : le chemin d'évolution pour la taille du pas à la génération $\mathrm{g}$.

$\mathrm{c}_{\sigma} \in[0,1]$ : la constante du temps pour l'adaptation de la taille du pas.

$\mu_{\mathrm{w}}$ : appelé variance masse de sélection effective (variance effective sélection mass).

Pour mettre à jour la matrice de covariance $\mathrm{C}$, il faut actualiser le chemin d'évolution pc, ça se fait suivant les équations suivantes :

$$
\begin{aligned}
\mathbf{p}_{c}^{(g+1)} & =\left(1-c_{c}\right) \mathbf{p}_{c}^{(g)}+h_{\sigma} \sqrt{c_{c}\left(2-c_{c}\right)} \sqrt{\mu_{u}} \frac{\mathbf{m}^{(g+1)}-\mathbf{m}^{(g)}}{\sigma^{(g)}} \\
& =\left(1-c_{c}\right) \mathbf{p}_{c}^{(g)}+h_{\sigma} \sqrt{c_{c}\left(2-c_{c}\right)} \sqrt{\mu_{w}} \sum_{i=1}^{\mu} w_{i}\left(\mathbf{C}^{(g)}\right)^{\frac{1}{2}} \mathbf{z}_{i: \lambda}
\end{aligned}
$$


Alors que la matrice de covariance devient comme suit :

$$
\begin{aligned}
C^{(g+1)}= & \left(1-c_{1}-c_{u}\right) \mathbf{C}^{(g)}+c_{1}\left(\mathbf{p}_{c}^{(g+1)}\left(\mathbf{p}_{c}^{(g+1)}\right)^{\mathrm{T}}+\delta\left(h_{\sigma}\right) \mathbf{C}^{(g)}\right) \\
& +c_{\mu} \sum_{i=1}^{u} w_{i}\left(\left(\mathbf{C}^{(g)}\right)^{\frac{1}{2}} \mathbf{z}_{i, \lambda}\right)\left(\left(\mathbf{C}^{(g)}\right)^{\frac{1}{2}} \mathbf{z}_{i: \lambda}\right)^{T}
\end{aligned}
$$

Avec : $\mathrm{p}_{\mathrm{c}}{ }^{(\mathrm{g})} \in \mathrm{R}^{\mathrm{d}}$ : le chemin d'évolution pour la taille du pas à la génération $\mathrm{g}$, sa valeur initiale est $\mathrm{pc}(0)=0 \mathrm{cc} \in[0,1]$ : la constante d'adaptation de la matrice de covariance. $\mathrm{h}_{\sigma}$ : est la fonction d'Heaviside. $c_{1}$ et $c_{\mu}$ : exprime le taux d'apprentissage (learning rate) respectivement pour rank-oneupdate et rank- $\mu$-update $\left.\mathrm{C}^{(t)}\right)^{\frac{1}{2}}$, leurs valeurs par défaut sont $c_{1}=\frac{2}{(d+1.3)^{2}+\mu_{u v}}$, et $c_{u}=\min \left(1-c_{1}, 2 \frac{\mu_{v}-2+1 / \mu_{w}}{(d+2)^{2}+\alpha_{u} \mu_{w} / 2}\right)$

avec $\alpha \mu=2$.

$\delta\left(h_{\sigma}\right)=\left(1-h_{\sigma}\right) c_{c}\left(2-c_{c}\right)$. Toutes ces étapes peuvent s'organiser dans un algorithme, qui s'exécute en fonction des conditions de convergence choisis, suivant un ordre bien précis, l'algorithme se répète jusqu'à la satisfaction de la condition de convergence en retournant le meilleur individus. Toutes les étapes nécessaires de la méthode CMA-ES sont ordonnées suivant l'algorithme 1 [LOS 14][BEN 19] :

Algorithm 1 CMAES $(\lambda, \mu)$

Initialiser les paramètres de CMA-ES $C(0)=I, \sigma^{(0)}$ et les paramètres de sélection initialiser le vecteur moyen $m^{(0)}$; préciser les conditions de convergence ; le nombre de génération $g \leftarrow-0$

tant que " la condition de convergence n'est pas vérifiée " faire Générer une population de $\lambda$ individus selon la loi : $x g_{i+1}$

$\left.\sim \mathbf{m}^{(g)}+\sigma^{(g)} \mathcal{N} 0, C^{(g)}\right)$, for $i=1, \ldots, \lambda$

Evaluer la fonction coût de chacun de $\lambda$ individus.

Classer les meilleurs individus générés

Déterminer le vecteur moyen par mutation des $\mu$ meilleurs individus selon :

$\mathbf{m}^{(g+1)}=\sum_{i=1}^{\mu} \omega_{i} z_{i}^{(g+1)}$

Mettre à jour le chemin d'évolution $P_{(\sigma)}$

Mettre à jour la taille de pas $\sigma^{(g+1)}$

Mettre à jour le chemin d'évolution $P_{(c)}$

Mettre à jour la matrice de covariance $C^{(g+1)}$

fin tant que

Retourner les meilleurs individus $x^{g}{ }_{i}+1$

\section{Intégration de CMA-ES avec le modèle élément finis}

La modélisation thermique a permet de simuler le comportement thermique du transistor, en plus il permet d'identifier les paramètres de la géométrie représentant un grand impact sur la température du 
fonctionnement du HEMT. L'épaisseur du substrat (a), l'épaisseur de la couche de nucléation (b) et le développement (c), sont les trois paramètres (en $\mu \mathrm{m}$ ) qui ont un grand impact sur le comportement thermique du transistor. Le but est de minimiser la température de fonctionnement du HEMT en agissant sur ces paramètres, par suite la fonction objectif sera la température maximale du fonctionnement. Le problème d'optimisation est :

$$
\left\{\begin{array}{c}
\min (f(x))=\min \left(\mathrm{T}_{\max }\right) \\
90<a<150 \\
0.1<\mathrm{b}<1 \\
80<\mathrm{c}<140
\end{array}\right.
$$

Afin de résoudre ce problème d'optimisation, un coulage des deux modèles : thermique et statistique sera développé. Le modèle thermique est repensé par le modèle éléments finis développé sous le logiciel Comsol multiphisics, le modèle statistique est représenté par la méthode CMA-ES codée sous le logiciel Matlab. L'intégration des deux modèles se fait suivant l'organigramme de la figure 2.

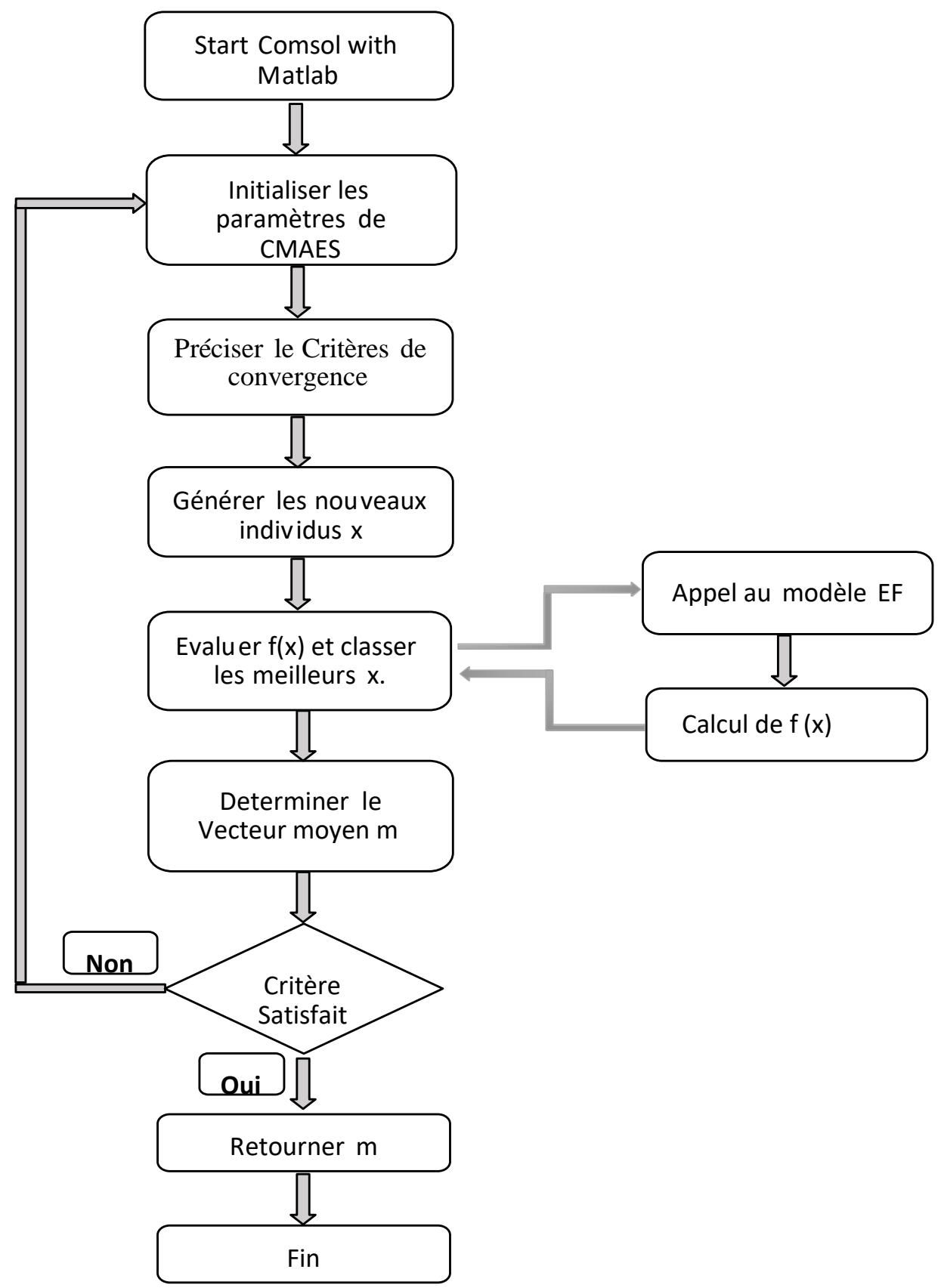

Figure 2. Organigramme de la méthode de l'optimisation avec CMAES. 
L'exécution de cet organigramme se fait par plusieurs étapes, il commence par l'initialisation des paramètres d'optimisation dans le code Matlab, les étapes suivants seront suivant l'algorithme 1, le calcul des images des individus s'effectue en appelant le modèle éléments finis sous le logiciel Comsol à travers une fonction codé sous Matlab. Quand le critère de l'arrêt cité dans l'initialisation est satisfait on obtient les valeurs optimums des paramètres choisis.

\section{Résultats numériques}

Après avoir exécuté le processus de l'optimisation, les résultats obtenus sont mentionné dans le tableau 5.2 : ce tableau contient les valeurs initiales et les valeurs optimales des paramètres choisis.

\begin{tabular}{|c|c|c|}
\hline Paramètres $(\mu \mathrm{m})$ & Valeurs initiales & Valeurs optimisées \\
\hline $\mathrm{a}$ & 0.8 & 0.153 \\
\hline $\mathrm{b}$ & 100 & 97.33 \\
\hline $\mathrm{c}$ & 100 & 119.13 \\
\hline
\end{tabular}

Tableau 2. Les paramètres de la géométrie du HEMT à optimiser

Le tableau suivant représente les valeurs initiales avant l'optimisation et les valeurs optimums après l'optimisation, pour observer l'influence de ces résultats sur le comportement thermique du transistor. On construit un nouveau modèle avec les paramètres géométriques optimums, des simulations numériques ont été effectuées en gardant les mêmes conditions de fonctionnement (conditions aux limites) et les autres paramètres constants. La figure 3 représente une comparaison de la distribution de la température dans les deux cas, sous une puissance dissipée de $5 \mathrm{w} / \mathrm{mm}$ et une température de référence de $50^{\circ} \mathrm{C}$ il apparait clairement que la distribution de la température est moins importante dans le deuxième cas ( $b$ ) que dans le premier cas (a). Donc la distribution de la température est améliorée après le processus de l'optimisation. 


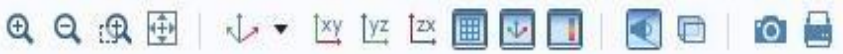

Max/min sur point: Température $(\operatorname{deg} \mathrm{C})$ Volume: Température (degC)

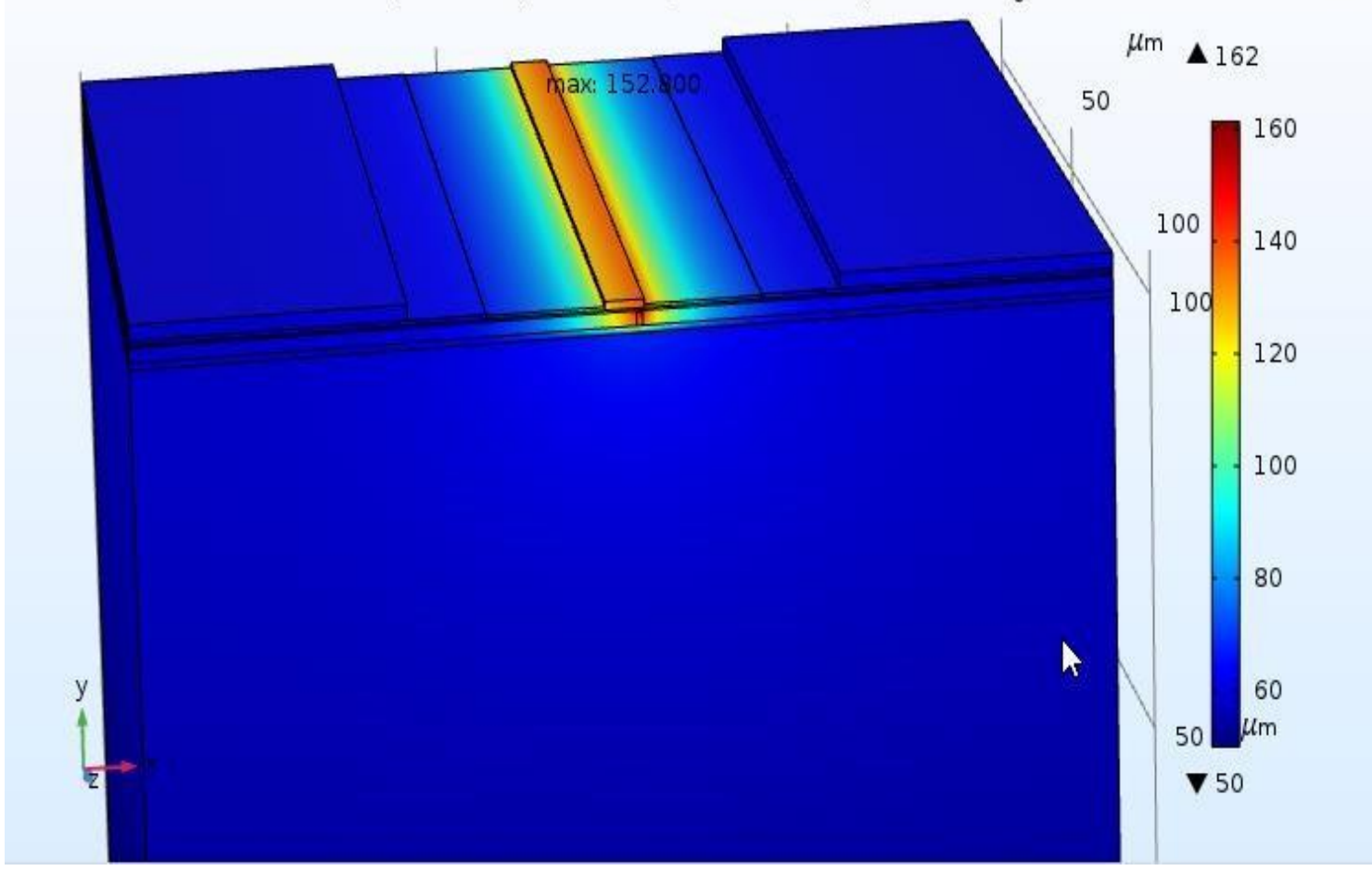

\section{A}

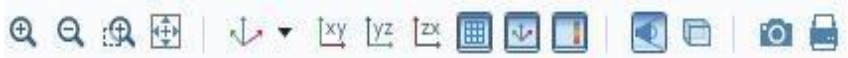

Max/min sur point: Température $(\mathrm{deg} C)$ Volume: Température $(\operatorname{deg} \mathrm{C})$

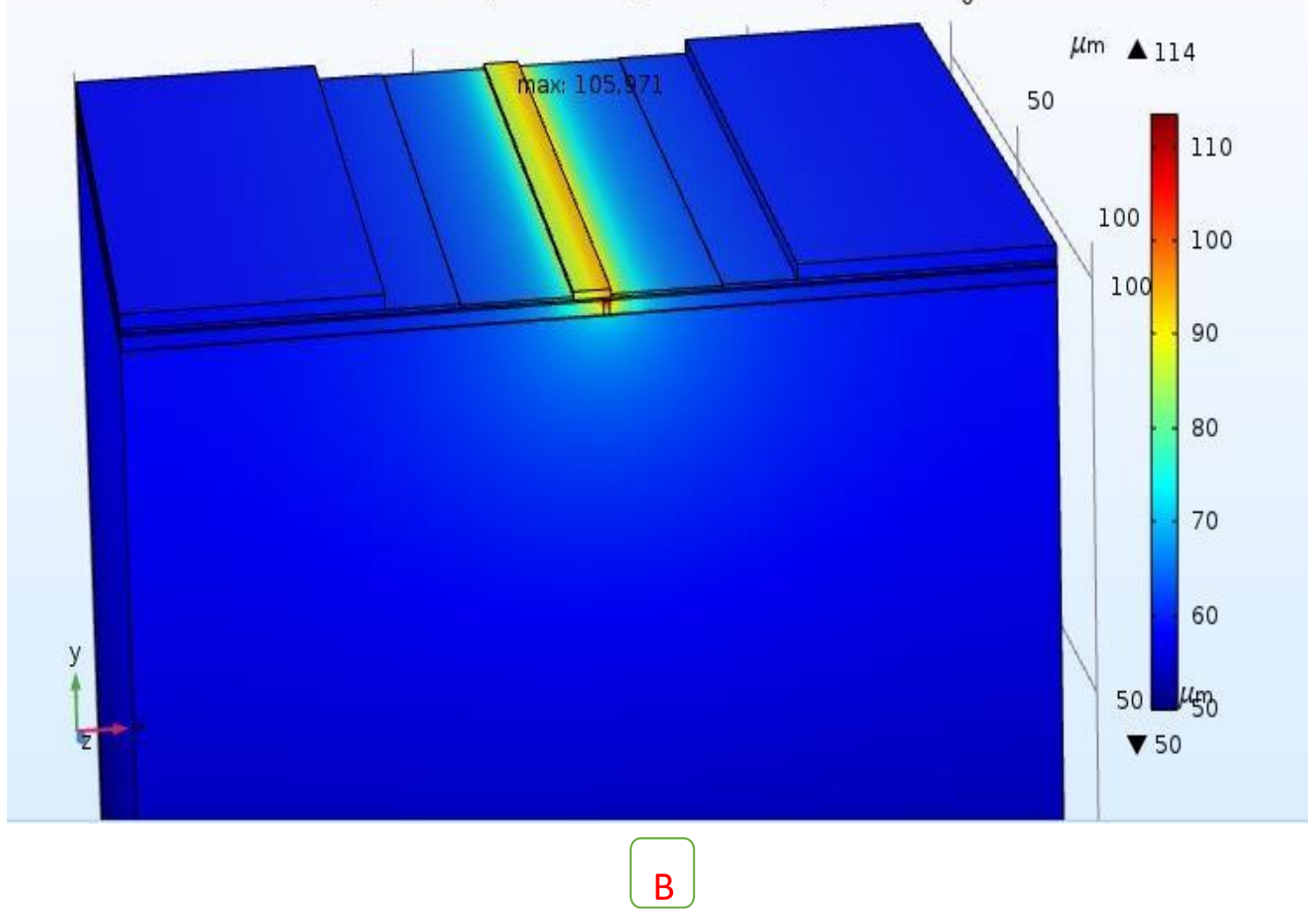

Figure 3. Distribution de la température au sien du transistor avant est après l'optimisation de la structure. 
De même, pour observer l'influence des valeurs optimums sur l'évolution de la température dans les différentes conditions du fonctionnement du HEMT, la figure 4 représente l'évolution de la température en fonction de la puissance dissipée dans les deux cas : avant et après l'optimisation, il est claire aussi que l'évolution de la température est moins importante après l'optimisation.

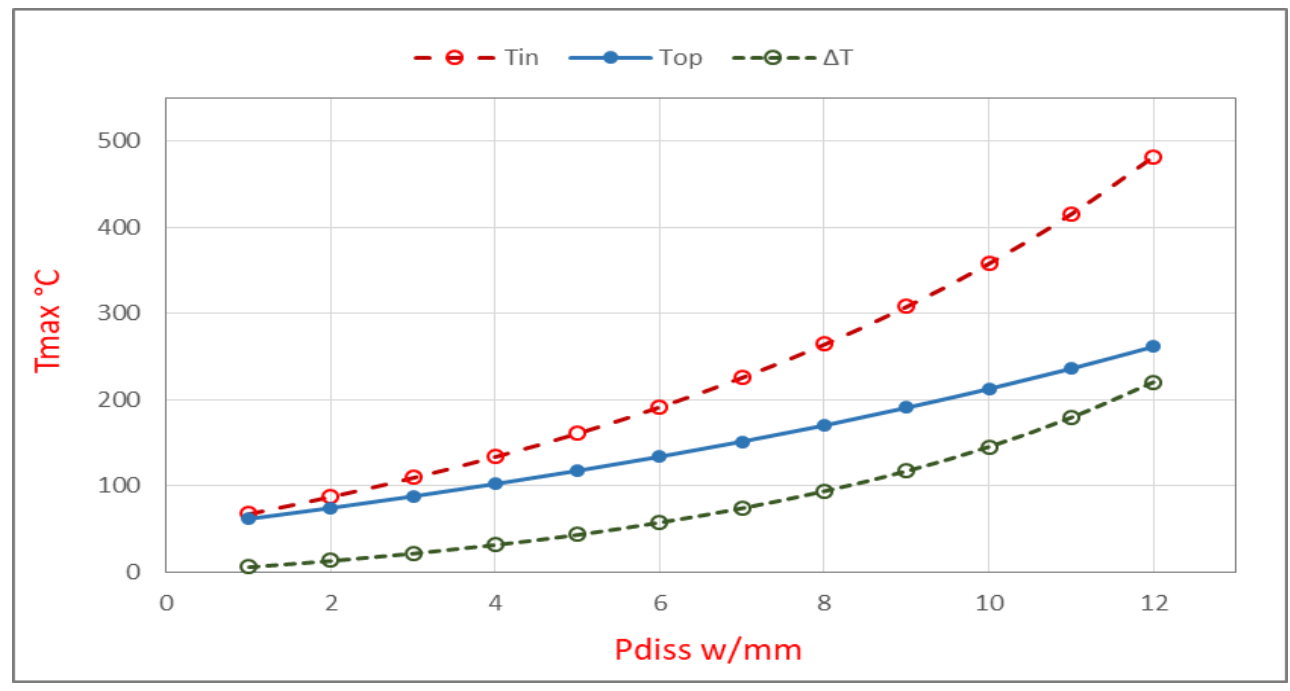

Figure 4. Evolution de la température du HEMT avant et après l'optimisation

Avant d'optimiser la structure du transistor sa température maximale du fonctionnement peut atteindre plus de $490{ }^{\circ} \mathrm{C}$, sous une puissance dissipée de $12 \mathrm{w} / \mathrm{mm}$ et une température de référence de $50^{\circ} \mathrm{C}$. Après avoir appliqué l'optimisation, la température maximale se limite à $270,35{ }^{\circ} \mathrm{C}$ dans les mêmes conditions du fonctionnement. On voit clairement aussi que la différence $\Delta T$ entre $T_{\text {in }}$ et $T_{o p}$ augmente en fonction de $P_{\text {diss. }}$

\section{Conclusion}

La fiabilité du HEMT est fortement en fonction de la température, la plupart de ses caractéristiques ont tendance à se dégrader avec l'augmentation de la température du fonctionnement, notamment la mobilité des électrons. D'autre part, la modélisation thermique par un modèle éléments finis développé sous le logiciel Comsol multiphysics a permis de simuler le comportement thermique du transistor et identifier les paramètres qui ont un grand impact sur ce comportement. Un modèle statistique de l'optimisation par la méthode CMAES est codé sous le logiciel Matlab, l'intégration de ce modèle avec le modèle thermique suivant un organigramme bien précis a permis d'optimiser la température maximale du HEMT, donc améliorer son comportement thermique et sa fiabilité. La méthode CMAES a montré une grande efficacité pour résoudre des problèmes de l'optimisation, mais elle est encore couteuse en termes de temps du calcul, ce qui nous ramène à penser à le minimiser par l'intégration d'autre méthode comme le metamodèle. Ce travail a pris en considération juste trois paramètres. Pour développer, il est préférable d'introduire autres paramètres et de réaliser une étude de fiabilité pour s'assurer de l'efficacité des résultats trouvés.

\section{Bibliographie}

[ELH 17] A. El hami, D. Delaux, and H. Grzeskowiak, Reliability of High-Power Mechatronic Systems 1: Aerospace and Automotive Applications: Simulation, Modeling and Optimization. ISTE éditions, 2017.

[GAR 16] S. García, I. Niguez-De-La-Torre, J. Mateos, T. González, and S. Pérez, "Impact of substrate and thermal boundary resistance on the performance of AlGaN/GaN HEMTs analyzed by means of electro-thermal Monte Carlo simulations," Semicond. Sci. Technol., vol. 31, no. 6, 2016, doi: 10.1088/0268-1242/31/6/065005. 
[WAN 18] L. Wang, J. Liu, W. Zhou, Z. Xu, Y. Wu, and H. Tao, "A novel method to dynamic thermal impedance and channel temperature extraction of GaN HEMTs," Int. J. Numer. Model. Electron. Networks, Devices Fields, no. October 2018, pp. 1-9, 2019, doi: 10.1002/jnm.2599.

[DYJ 18] D. Y. Jeon et al., "Effects of series resistance and interface properties on the operation of AlGaN/GaN high electron mobility transistors," Microelectron. Eng., vol. 199, pp. 40-44, 2018, doi: 10.1016/j.mee.2018.07.010.

[DON 19] Y. Dong, Z. Xie, D. Chen, H. Lu, R. Zhang, and Y. Zheng, "Effects of dissipative substrate on the performances of enhancement mode AlInN/GaN HEMTs," Int. J. Numer. Model. Electron. Networks, Devices Fields, vol. 32, no. 1, pp. 1-9, 2019, doi: 10.1002/jnm.2482.

[CHE 19] Y. Chen et al., "Improved quasi-physical zone division model with analytical electrothermal Ids model for AlGaN/GaN heterojunction high electron mobility transistors," Int. J. Numer. Model. Electron. Networks, Devices Fields, no. May, pp. 1-17, 2019, doi: 10.1002/jnm.2630.

[DAS 06] J. Das et al., "a Temperature Analysis of High-Power Algan / Gan Hemts," Dans Proc. 12th Int. Work. Therm. Investig. ICs - THERMINIC 2006, pp. 2-5, 2006.

[JIA 18] Y. Jia, Y. Xu, and Y. Guo, "A Universal Scalable Thermal Resistance Model for Compact Large-Signal Model of AlGaN/GaN HEMTs," IEEE Trans. Microw. Theory Tech., vol. 66, no. 10, pp. 4419-4429, 2018, doi: 10.1109/TMTT.2018.2854185.

[BEL 19] H. Belmabrouk et al., "Modeling the simultaneous effects of thermal and polarization in InGaN/GaN based high electron mobility transistors," Optik (Stuttg)., p. 163883, 2019, doi: 10.1016/j.ijleo.2019.163883.

[RAD 19] U. Radhakrishna, Physics-based III-Nitride device modeling, 1st ed., vol. 102. Elsevier Inc., 2019.

[SAM 20] S. Samira, "Modeling of Enhancement-Mode GaN-GIT Application,” IEEE Trans. Electron Devices, pp. 1-7, 2020.

[ALI 20] M. A. Alim, S. Afrin, A. A. Rezazadeh, and C. Gaquiere, "Thermal response and correlation between mobility and kink effect in GaN HEMTs," Microelectron. Eng., vol. 219, no. April 2019, p. 111148, 2020, doi: 10.1016/j.mee.2019.111148.

[BAC 15a] L. Baczkowski, "Modélisation et Caractérisation Thermique de Transistors de Puissance Hyperfréquence GaN et Conséquences sur la Fiabilité de Modules Radars d'Émission/Réception en Bande X," Université de Lille, 2015.

[HAN 16] N. Hansen, “The CMA Evolution Strategy : A Tutorial arXiv : 1604 . 00772v1 [ cs . LG ] 4 Apr 2016,” 2016.

[HAM 19a] H. Hamdani, B. Radi, and A. El Hami, "Metamodel assisted evolution strategies for global optimization of solder joints reliability in embedded mechatronic devices," Microsyst. Technol., vol. 25, no. 10, pp. 3801-3812, 2019, doi: 10.1007/s00542-019-04520-1.

[AMA 19] A. Amar, B. Radi, and A. El Hami, "La modélisation thermique de transistor a haute puissance de type HEMT," Incert. fiabilité des systèmes multiphysiques, vol. 3, no. 2019, pp. 1-7, 2019, doi: 10.21494/iste.op.2019.0431.

[BAC 15b] L. Baczkowski et al., "Temperature measurements in RF operating conditions of AlGaN/GaN HEMTs using IR microscopy and Raman spectroscopy,” Eur. Microw. Week 2015 , vol. 5, pp. 152-155, 2015, doi: 10.1109/EuMIC.2015.7345091.

[ELH 15] A. El Hami and P. Pougnet, Embedded Mechatronic Systems 2: Analysis of Failures, Modeling, Simulation and Optimization. ISTE éditions, 2015.

[JAR 14] O. Jardel et al., "InAIN/GaN HEMTs based L-band high-power packaged amplifiers," Int. J. Microw. Wirel. Technol., vol. 6, no. 6, pp. 565-572, Dec. 2014, doi: 10.1017/s175907871400004x.

[CHE 13] S. Cheng and P. C. Chou, "Novel packaging design for high-power GaN-on-Si high electron mobility transistors (HEMTs),” Int. J. Therm. Sci., vol. 66, pp. 63-70, Apr. 2013, doi: 10.1016/j.ijthermalsci.2012.10.003.

[21] R. Simon and W. Monika, "Identi cation des propriétés hygrothermiques des matériaux par stratégie d ' évolution CMA," in Conférence IBPSA France-Arras-2014 Identi?cation, 2014, pp. 1-8.

[HAM 19b] H. Hamdani, B. Radi, and A. El Hami, "Optimization of solder joints in embedded mechatronic systems via Kriging-assisted CMA-ES algorithm," Int. J. Simul. Multidiscip. Des. Optim., vol. 10, p. A3, 2019, doi: $10.1051 / \mathrm{smdo} / 2019002$.

[HAN 17] N. Hansen, "Thèse de doctorat de 1 ' Université Paris-Saclay préparée à 1 ' Université Paris-Sud Ecole doctorale n 580 Sciences et Technologies de 1 ' Information et de la Communication par M . Ouassim AIT ELHARA Stochastic Black-Box Optimization and Benchmarking in ," 2017. 
[SIG 13] O. Sigaud and F. Stulp, "Adaptation de la matrice de covariance pour 1 ' apprentissage par renforcement direct," pp. 243-263, 2013, doi: 10.3166/RIA.27.243-263.

[LOS 14] I. Loshchilov, “A Computationally Efficient Limited Memory CMA-ES for Large Scale Optimization,” 2014.

[BEN 19] E. Benhamou, J. Atif, R. Laraki, E. Benhamou, J. Atif, and R. Laraki, "A discrete version of CMA-ES To cite this version : HAL Id : hal-02011531 A discrete version of CMA-ES,” 2019. 